\title{
External validation of a clinical pharmacy intervention in geriatric inpatients: a controlled study
}

\author{
Lorenz Van der Linden ${ }^{1,2}$ (1) $\cdot$ Liesbeth Decoutere ${ }^{3} \cdot$ Leen Beerten $^{3} \cdot$ Toon Delva $^{4} \cdot$ Isabel Spriet $^{1,2} \cdot$ Johan Flamaing $^{5,6}$. \\ Jos Tournoy ${ }^{5,6}$
}

Received: 27 June 2018 / Accepted: 9 May 2019

(c) Springer Nature Switzerland AG 2019

\begin{abstract}
Background Clinical pharmacist interventions have been shown to improve drug use in older adults. Study findings are seldom externally validated however. Objective First, to validate a minimized iteration of a previously tested intervention of clinical pharmacists in a non-academic setting with limited staffing resources. Second, to compare the potentially inappropriate medication (PIM) reduction to two previous controlled interventional studies. Methods A controlled study was performed at geriatric wards. The control group received usual care. The intervention group was exposed to a clinical pharmacist led medication review, based on the use of the RASP list, (the Rationalization of Home Medication by an Adjusted STOPP list in Older Patients). Drug use on admission and at discharge were evaluated, including the number of RASP-identified potentially inappropriate medications (PIMs). The PIM reduction was compared to two previous controlled study findings using a linear mixed model. Results Drug use declined during hospital stay, without differences between control $(\mathrm{n}=29)$ and intervention group $(n=32)$. Antidepressants and hypnotic drugs were discontinued more frequently in IG patients. More PIMs were reduced in the intervention patients (control vs. intervention: 1.0 vs. 3.0, $p<0.001$ ). Across three controlled studies, a robust reduction of 1.56 PIMs (95\% confidence interval 1.10-2.02, $p<0.001$ ) was observed in favor of the CP interventions. Conclusion The minimal CP intervention resulted in fewer RASP PIMs. No net reduction of drug use was observed, yet fewer antidepressants and hypnotic drugs were used. The RASP PIM reduction was comparable to previous investigations.
\end{abstract}

Keywords Belgium · Clinical pharmacy · External validation · Minimal intervention · Older inpatients · Polypharmacy · Potentially inappropriate medication

\section{Impacts on Practice}

Lorenz Van der Linden

lorenz.vanderlinden@uzleuven.be

1 Pharmacy Department, University Hospitals Leuven, Herestraat 49, 3000 Louvain, Belgium

2 Department of Pharmaceutical and Pharmacological Sciences, KU Leuven, Louvain, Belgium

3 Pharmacy Department, Jessa Hospital, Hasselt, Belgium

4 Department of Geriatric Medicine, Jessa Hospital, Hasselt, Belgium

5 Department of Geriatric Medicine, University Hospitals Leuven, Louvain, Belgium

6 Department of Chronic Diseases, Metabolism and Ageing, KU Leuven, Louvain, Belgium
- When confronted with limited staffing resources, clinical pharmacists can still impact drug use in geriatric inpatients by applying a minimal clinical pharmacy intervention.

- A minimal clinical pharmacy intervention, characterized by a limited ward-based presence ( $<30 \mathrm{~min} \cdot$ per patient), was associated with fewer potentially inappropriate medications at discharge.

- The reduction in the number of potentially inappropriate medications is consistent across three different settings. 


\section{Background}

Drug-related problems (DRP) remain prevalent in older inpatients [1]. The high prevalence can partially be explained by a multitude of conditions being managed pharmacologically [2]. Consequentially, high rates of polypharmacy and potentially inappropriate medication (PIM) use can be observed in multimorbid older adults, both of which can exacerbate clinically relevant drugrelated harm [3].

Ward-based clinical pharmacy services might decrease the burden associated with this drug-related harm [3]. Clinical pharmacists (CP) have been shown to positively impact the overall appropriateness of a drug regimen, by decreasing the amount of DRP or PIMs [4]. Spinewine et al. [5] showed that a CP intervention in geriatric inpatients was associated with an improvement in drug therapy appropriateness, in terms of the Medication Appropriateness Index (odds ratio: 9.1, 95\% confidence interval (CI) 4.2-21.6). Gillespie et al. found that a CP intervention resulted in a detection of 476 DRP in 182 older inpatients, $75 \%$ of which were resolved as a result of the pharmacist's recommendations [6]. In addition, clinical outcomes, such as unplanned readmissions, might be improved as well by a comprehensive CP intervention [7]. This was observed by Gillespie et al. [6], who inferred a reduced risk for the number of hospital visits (relative risk: $0.85,95 \% \mathrm{CI}$ $0.72-0.99$ ) in a predominantly octogenarian population. This clinical impact of a CP intervention has however not been robustly confirmed yet in any meta-analysis $[8,9]$. Furthermore, the external validity of published findings remains limited as not all hospitals have access to or have the necessary financial resources to support ward-based CP services, which might limit downstream uptake [10]. For example, in Belgium there is on average one hospital pharmacist available per 150 beds to perform the routine pharmacy tasks, with additional governmental funding to support CP services at a rate of 0.25 full-time equivalent (FTE) per 250 beds [11]. In comparison, a more comprehensive CP intervention such as described by Gillespie et al. and Ravn-Nielsen et al. required on average $2 \mathrm{~h}$ per patient, which is very difficult to implement in such a setting $[6,12]$. There is hence a need for more data on the use of directed, or 'minimal', pharmaceutical interventions to facilitate further implementation of ward-based $\mathrm{CP}$ services in the hospital setting [13].

In a previous investigation, performed in a large academic hospital, we showed that an extensive CP intervention decreased the number of PIMs at discharge in older inpatients [1]. Clinical pharmacists based their recommendations in a non-exhaustive manner on the RASP (Rationalization of Home Medication by an Adjusted STOPP list in Older Patients) list [14]. The RASP list was developed in a large teaching hospital located in Belgium [14]. It was conceptualized as an explicit screening tool for PIMs and it was aimed to have an add-on value compared to the first version of the Irish Screening Tool of Older Person's Prescriptions, in particular regarding very old inpatients. Its use and content were subsequently validated among Belgian experts on geriatric pharmacotherapy. The second validated version of the RASP criteria consisted of $76 \mathrm{cri}-$ teria, distributed over 12 chapters.

In this short report, the findings of a follow-up controlled study are presented, which took place at geriatric wards in a non-academic hospital and in which a minimal rendition of a RASP-based intervention was investigated.

\section{Aim of the study}

The study sought to externally validate a minimized iteration of a previously tested RASP-based CP intervention, in a non-academic hospital setting with limited staffing resources. Furthermore, we aimed to compare the observed RASP PIM reduction to the results attained in two previous studies.

\section{Ethics approval}

The study was approved by the hospital's ethics committee (approval number: 13.91/ger13.02).

\section{Methods}

\section{Study design, setting and trial participants}

A study with a prospective, controlled single-blinded design was undertaken at two acute geriatric care wards of the Jessa Hospital, a 1000-bed non-academic hospital, situated in Hasselt, Belgium. Study design and criteria for trial participation were adopted from the original study [1]. Patients were consecutively screened for study eligibility when research pharmacists were present at the wards. In sum, Dutch-speaking geriatric patients who were admitted from home or a nursing home and who gave oral and written consent were eligible for study participation. Geriatric patients in whom all drug therapies were actively withdrawn due to terminal illness were excluded, as well as those who were not discharged back to their home or nursing home. 


\section{Usual care and intervention}

Control patients received usual geriatric care and a minimal CP intervention was provided in intervention patients in addition to usual geriatric care. Usual care consisted of normal geriatric care, provided by a senior geriatrician. In the intervention group, the RASP list was applied by two trained CP (LD and LB). The study was mainly performed by a hospital pharmacist-in-training (LB). A pharmacistin-training is a federally registered pharmacist (5-year program) who has been allowed to pursue an additional Master degree in Hospital Pharmacy (3-year program). Recommendations were provided directly to the prescribing physicians [14]. The intervention was considered to be minimal, opposed to the previously investigated intervention, owing to the limited ward-based presence of the involved pharmacists and the limited time per patient [1]. In this study, the intervention was also exclusively based on the use of the RASP list. The pharmacy department agreed to exempt two CP (LD, LB) from daily hospital pharmacy routine (e.g. distribution or compounding) for a combined total of $3 \mathrm{~h}$ per week, during which patients were enrolled and medication reconciliation and review were performed. Consequentially, pharmacists had approximately 30 min per patient at their disposal to perform all activities. In addition, contrary to the previous investigations (RASP LEUVEN, RASP IGCT) which were set in teaching hospital, no physicians-in-training were involved, only senior geriatricians $[1,15]$.

\section{Data collection}

Weight (kg), age (years), the number of preadmission drugs and creatinine clearance $(\mathrm{CrCl}$, according to the CockcroftGault equation) were determined upon admission to the wards. The following data was collected as well: length of stay (days), number of drugs at discharge, as well as the number and type of RASP-identified potentially inappropriate medications (i.e. RASP PIM) on admission and at discharge.

\section{Outcome measures}

The primary outcome measure was the RASP PIM reduction during hospital stay. Secondary outcome measures were the acceptance rate of the provided recommendations by the ward-based physicians in the intervention group, the number of discontinued preadmission drugs, the total number of discontinued drugs (from admission to discharge), the number of newly initiated drugs, and also the involved drug classes (defined at the Anatomical Therapeutic Chemical
(ATC) classification 4 level) that were initiated or discontinued during hospital stay.

\section{Sample size estimation}

Sample size was originally estimated to detect a mean difference of 2.6 RASP PIMs (standard deviation (SD): 2.0) from admission to discharge, as observed in the intervention arm of the original RASP study, with $99 \%$ power and a type I error of $1 \%$ [1]. Considering a potential $20 \%$ loss due to attrition, a total of 24 study subjects had to be enrolled in the intervention arm. If in total $48(=2 \times 24)$ patients were enrolled, this would also provide sufficient power (80\%) to detect a mean difference of at least 1.1 RASP PIMs between the control and intervention group.

\section{Statistical analysis}

Normality was assessed using the Kolmogorov-Smirnov test and by manually evaluating the histograms. The Mann-Whitney test was used to compare unrelated nonparametric continuous variables and the Wilcoxon signed rank test to compare related continuous variables. The Fisher Exact test was chosen to compare proportions.

An exploratory post hoc analysis was performed, comparing the results on RASP PIM reduction from admission to discharge of RASP JESSA with those from previous studies (RASP LEUVEN and RASP IGCT) [1, 15]. A linear mixed model was used to identify potential relationships between the dependent outcome RASP PIM reduction and the covariates 'group' (control vs. intervention) and 'study' (RASP JESSA, RASP LEUVEN, RASP IGCT), while verifying whether an interaction existed between group and study. In the absence of a statistically significant group $\mathrm{x}$ study interaction, main effects results were reported.

Statistical significance was set at $p<0.05$ (two-tailed). IBM SPSS Statistics for Windows version 25.0 was used for statistical analysis (IBM Corporation, Armonk, NY, USA). $\mathrm{G}^{*}$ Power 3.1 was used to determine the sample size.

\section{Results}

In total 61 (control: 29, intervention: 32 ) of the 321 eligible patients were enrolled from January to March 2014. No differences were found in any of the following characteristics between control and intervention patients. Among all patients, the median age was 86 years (IQR: $80-91$ ) and length of stay of 12 days (IQR: 7-19). Upon admission, patients weighed on average $65 \mathrm{~kg}$ (IQR: 55-75), used on average 10 drugs (IQR: $8-13$ ) and had moderate to severe renal impairment (CrCl: $34.9 \mathrm{ml} / \mathrm{min}$, IQR: 24.3-51.4). 
No significant difference was seen regarding the reduction of the number of preadmission drugs per patient (control: 3.5 (IQR: 2.0-7.3); intervention: 5.0 (IQR: 4.0-6.0); $p=0.160)$. The total number of drugs per patient declined during hospital stay in both groups (admission vs. discharge: 10 (IQR: 8-13) vs. 9 (IQR: 6-11), $p<0.001$ ), without significant differences between control and intervention groups $(p=0.319)$. More antidepressants were discontinued in the intervention group (control vs. intervention: 10.0 vs. $35.5 \%$, $p=0.031)$, as well as more hypnotic agents (6.7 vs. $29.0 \%$, $p=0.043$ ).

No significant differences were seen concerning the number of newly initiated drug therapies (control vs. intervention: 2.0 (IQR: $1.0-3.0$ ) vs. 3.0 (IQR: $2.0-4.0$ ), $p=0.091$ ). Significant differences were observed for the following specific drug classes at the ATC4 level: angiotensin-converting enzyme inhibitors and angiotensin receptor blockers combined (control vs. intervention: 3.3 vs. $25.8 \%, p=0.026$ ), and calcium-vitamin D (20.0 vs. $54.8 \%, p=0.008)$.

In the control group, a reduction of RASP PIMs was observed from 3.0 (IQR: 1.0-6.0) to 2.0 (IQR: 1.0-3.5) $(p=0.002)$. In the intervention group, RASP PIMs were reduced from a median of 3.0 (IQR: 2.0-5.0) on admission to 0.0 (IQR: $0.0-1.0)$ at discharge $(p<0.001)$. Acceptance rate of CP recommendations was $90.1 \%$. More RASP PIMs were stopped during hospital stay in the intervention group

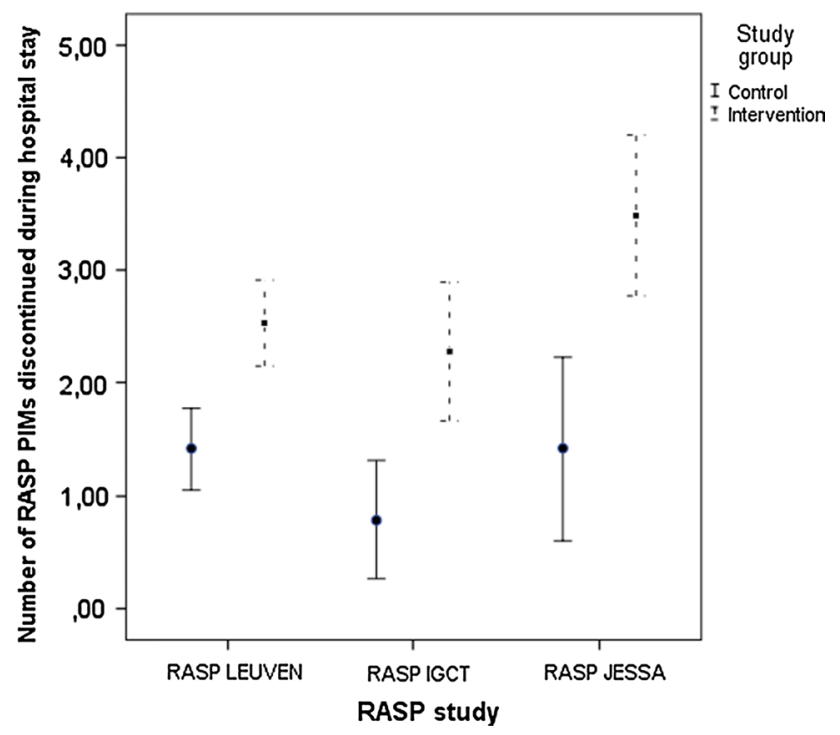

Fig. 1 RASP PIM Reduction Across Three Controlled Investigations. The panel shows the mean number (and the 95\% confidence interval) of discontinued RASP PIMs during hospital stay. Per study, data are shown for the control and intervention arms. Data from three studies are depicted: RASP LEUVEN [1], RASP IGCT LEUVEN [14], RASP JESSA. RASP (Rationalization Of Home Medication By An Adjusted STOPP in Older Patients), PIM (potentially inappropriate medications) (control vs. intervention: 1.0 (IQR: 0.0-3.0) vs. 3.0 (IQR: 2.0-5.0), $p<0.001)$.

RASP PIM reduction did not differ significantly between RASP JESSA, RASP LEUVEN and RASP IGCT (see Fig. 1). No statistically significant interaction was found between group (control vs. intervention) and study (RASP JESSA vs. RASP LEUVEN vs. RASP IGCT) $(p=0.186)$. Independently of the individual investigations, the main effects analysis showed that more RASP PIMs were discontinued in the intervention group (mean difference: $1.56,95 \%$ CI 1.10-2.02, $p<0.001)$.

\section{Discussion}

A CP intervention was developed and implemented, building on previous experiences [1]. Central to the previous approach was the semi-permanent ward-based presence of a trained CP, who furthermore was not limited to using the RASP list as basis for the intervention. In contrast, the novelty of the current approach resided within the intervention being a minimized iteration which was mainly carried out by a hospital pharmacist-in-training, who furthermore relied solely on the RASP list. Our results suggest that such an intervention can successfully be deployed, using a limited amount of time (i.e. $<3 \mathrm{~h}$ per week).

The approach of such a minimal intervention may become exceedingly relevant as there are only limited available resources for hospital boards to allocate to drug improvement services. The recent advent of improved clinical decision support systems will probably make way for an increasing uptake of back-office CP services [13, 16]. These probably cost-effective, yet still clinically unproven (in terms of harder clinical endpoints such as readmissions), services might limit future uptake of more front-office inclined services [16]. Hence, the results provided here can be used to support and importantly initiate ward-based services, even within a limited mandate. Yet, a larger time investment, not a smaller, will most likely be needed to impact clinical outcome $[4,12]$.

Our study also adds to the current body of evidence that a PIM reduction can be attained during hospital stay. This falls in line with a recent report of Sennesael et al. [17]. Their results suggested that a ward-based presence of a pharmacist should be recommended to provide sufficient impetus for a medicines optimization intervention to fully work. The specific classes of drugs that were targeted the most were furthermore comparable to previous experiences with the RASP list, with antidepressants and hypnotic agents being among the most frequently discontinued medications [1].

The findings of the current study further underline the utility of the RASP list as an educational aid to assist healthcare providers. Comparable RASP PIM reductions were 
observed in the three intervention arms on a background of usual geriatric care $[1,15]$. The intervention delivery differed between the three investigations: in the RASP LEUVEN trial a quasi-permanent ward-based presence was guaranteed while in the RASP IGCT and the current RASP JESSA this was much more limited. Usual geriatric care differed as well, with senior geriatricians being the main prescribers in the current study, as opposed to previous investigations, where residents were the main prescribers $[1,15]$.

We believe that the results of this pilot study are valid due to its controlled, prospective and single-blinded design, with few selection criteria. Furthermore, acceptance rate was high and our results are in line with our own previous findings $[1,15]$. Conversely, several limitations should be taken into account, such as the limited availability of the CP team and the lack of resources, which led to an overall small sample size. This was in part compensated for by performing a sample size calculation a priori to primarily ensure excellent power to detect the before/ after RASP PIM reduction in the intervention arm. This also provided sufficient power, albeit secondary, to detect a difference in RASP PIM reduction between control and intervention groups. Also, patient characteristics were only documented in a limited manner, potentially confounding our results. Nonetheless, no differences were observed regarding RASP PIM reduction among the three controlled investigations. Furthermore, no patient-related outcomes or clinical outcome were determined within the study. This should be targeted in future studies, that should aim to improve drug use and as a result clinical outcome, while bearing in mind limited resources [10].

\section{Conclusion}

A minimized iteration of a RASP-based clinical pharmacy intervention was associated with a larger PIM reduction in geriatric inpatients compared to usual care. No net reduction of drug use was observed, yet fewer antidepressants and hypnotic drugs were associated with the intervention. The PIM reduction was comparable to previous investigations withg the use of the RASP tool.

Acknowledgements We wish to acknowledge Mark Lutin for his collaboration to the study as well as all the nurses, patients and their caretakers.

Funding No specific funding was received to perform this study (design, execution). Isabel Spriet and Lorenz Van der Linden received a partial research scholarship of the University Hospitals Leuven, Leuven, Belgium.

Conflicts of interest There are no conflicts of interest to disclose.

\section{References}

1. Van der Linden L, Decoutere L, Walgraeve K, Milisen K, Flamaing J, Spriet I, Tournoy J. Combined use of the rationalization of home medication by an adjusted STOPP in older patients (RASP) list and a pharmacist-led medication review in very old inpatients: impact on quality of prescribing and clinical outcome. Drugs Aging 2017;34(2):123-33.

2. Boyd CM, Darer J, Boult C, Fried LP, Boult L, Wu AW. Clinical practice guidelines and quality of care for older patients with multiple comorbid diseases: implications for pay for performance. JAMA 2005;294(6):716-24.

3. Spinewine A, Schmader KE, Barber N, Hughes C, Lapane KL, Swine C, Hanlon JT. Appropriate prescribing in elderly people: how well can it be measured and optimised? Lancet 2007;370(9582):173-84.

4. Gillespie U, Alassaad A, Henrohn D, Garmo H, HammarlundUdenaes M, Toss H, Kettis-Lindblad A, Melhus H, Morlin C. A comprehensive pharmacist intervention to reduce morbidity in patients 80 years or older: a randomized controlled trial. Arch Intern Med. 2009;169(9):894-900.

5. Spinewine A, Swine C, Dhillon S, Lambert P, Nachega JB, Wilmotte L, Tulkens PM. Effect of a collaborative approach on the quality of prescribing for geriatric inpatients: a randomized, controlled trial. J Am Geriatr Soc. 2007;55(5):658-65.

6. Gillespie U, Alassaad A, Henrohn D, Garmo H, HammarlundUdenaes M, Toss H, Kettis-Lindblad A, Melhus H, Morlin C. A comprehensive pharmacist intervention to reduce morbidity in patients 80 years or older: a randomized controlled trial. Arch Intern Med. 2009;169(9):894-900.

7. Kaboli PJ, Hoth AB, McClimon BJ, Schnipper JL. Clinical pharmacists and inpatient medical care: a systematic review. Arch Intern Med. 2006;166(9):955-64.

8. Christensen M, Lundh A. Medication review in hospitalised patients to reduce morbidity and mortality. Cochrane Database Syst Rev. 2016;2:8986.

9. Renaudin P, Boyer L, Esteve MA, Bertault-Peres P, Auquier P, Honore S. Do pharmacist-led medication reviews in hospitals help reduce hospital readmissions? A systematic review and meta-analysis. Br J Clin Pharmacol. 2016;82(6):1660-73.

10. Van der Linden L, Hias J, Walgraeve K, Flamaing J, Spriet I, Tournoy J. Clinical pharmacy services on geriatric care wards: catch 22 of implementation and research. Drugs Aging 2018;35(5):375-7.

11. Somers A, Spinewine A, Spriet I, Steurbaut S, Tulkens P, Hecq JD, Willems L, Robays H, Dhoore M, Yaras H, Vanden Bremt I, Haelterman M. Development of clinical pharmacy in Belgian hospitals through pilot projects funded by the government. Acta Clin Belg. 2018;30:1-7.

12. Ravn-Nielsen LV, Duckert ML, Lund ML, Henriksen JP, Nielsen ML, Eriksen CS, Buck TC, Pottegard A, Hansen MR, Hallas J. Effect of an in-hospital multifaceted clinical pharmacist intervention on the risk of readmission: a randomized clinical trial. JAMA Intern Med. 2018;178(3):375-82.

13. Bos JM, van den Bemt PM, Kievit W, Pot JL, Nagtegaal JE, Wieringa A, van der Westerlaken MM, van der Wilt GJ, de Smet PA, Kramers C. A multifaceted intervention to reduce drugrelated complications in surgical patients. Br J Clin Pharmacol. 2017;83(3):664-77.

14. Van der Linden L, Decoutere L, Flamaing J, Spriet I, Willems L, Milisen K, Boonen S, Tournoy J. Development and validation of the RASP list (rationalization of home medication by an adjusted STOPP list in older patients): a novel tool in the management of geriatric polypharmacy. Eur Geriatr Med. 2014;5(3):175-80. 
15. Van der Linden L, Hias J, Dreessen L, Milisen K, Flamaing J, Spriet I, Tournoy J. Medication review versus usual care to improve drug therapies in older inpatients not admitted to geriatric wards: a quasi-experimental study (RASP-IGCT). BMC Geriatr. 2018;18(1):155.

16. Dalton K, O'Brien G, O'Mahony D, Byrne S. Computerised interventions designed to reduce potentially inappropriate prescribing in hospitalised older adults: a systematic review and meta-analysis. Age Ageing 2018;47(5):670-8.

17. Sennesael AL, Dalleur O, Henrard S, Artoisenet C, Schoevaerdts $\mathrm{D}$, Spinewine A. Implementing a screening tool to improve prescribing in hospitalized older patients: a pilot study. Int J Clin Pharm. 2018;40(1):15-9.

Publisher's Note Springer Nature remains neutral with regard to jurisdictional claims in published maps and institutional affiliations. 